\title{
Is the B[e] Star V2028 Cyg a Binary?
}

\author{
Jan Polster ${ }^{1,2}$, Daniela Korčáková ${ }^{2}$, Viktor Votruba $^{1,3}$, Petr Škoda ${ }^{3}$, \\ Miroslav Šlechta ${ }^{3}$ and Blanka Kučerová ${ }^{1}$ \\ ${ }^{1}$ Faculty of Science, Masaryk University, Kotlářská 2, 61137 Brno, CZ \\ ${ }^{2}$ Astronomical Institute, Charles University, V Holešovičkách 2, 18000 Praha 8, CZ \\ ${ }^{3}$ Astronomical Institute of the AV ČR, Fričova 298, 25165 Ondřejov, CZ \\ email: polster@physics.muni.cz, kor@sirrah.troja.mff.cuni.cz
}

\begin{abstract}
V2028 Cyg shows a B[e] phenomenon. Due to the presence of both cool (K7III) and hot (B4III) components in the spectra, it is supposed to be a binary. Our modelling of the time variability of $\mathrm{H} \alpha$ line bisectors shows, however, that this hybrid spectrum can originate in one star, which is surrounded by a disc.
\end{abstract}

Keywords. stars: emission-line, B[e], binaries: spectroscopic, stars: individual (V2028 Cyg)

\section{Introduction}

V2028 Cyg is a star showing the B[e] phenomenon, i.e. lines from the forbidden transitions are present in its spectra. This is indicative of a very extended circumstellar envelope. Due to this reason, the nature of this object is unknown. The determination of the stellar parameters is very uncertain, since the commonly used synthetic spectra are not applicable. Therefore, we focused on a study of time dependencies of the spectral features. Our aim was also to better specify the role of the binarity in this system. The spectrum of V2028 Cyg is very atypical among stars with the B[e] phenomenon, since it is composed of two kinds of spectra: K7III and B4III (Zickgraf 2001; Bergner et al. 1995).

We obtained a series of 88 spectra (Polster et al. 2010; Polster et al. 2011a,b) from the Ondřejov $2 \mathrm{~m}$ telescope in the spectral range 6250-6770 $\mathrm{A}$ ( $\mathrm{H} \alpha$ line, $\mathrm{R} \sim 12500$ ). These data were used for the description of the spectral-line variability. The time dependencies of equivalent width, line intensities, radial velocities and bisectors of the $\mathrm{H} \alpha$ line were modelled.

\section{Modelling}

In order to narrow the set of possible geometries, we constructed a simple numerical model. We assumed an optically thin medium, which allows us to add (subtract) radiation along the line-of-sight. Another simplification was a Gaussian profile of the line in every cell. The grid was defined with respect to the velocity field. Such a model is too simple for the $\mathrm{B}[\mathrm{e}]$ star description, however, it can be used in this case. We do not derive the parameters of the system, but we only compare the relative time variations of certain quantities - equivalent width, line intensities, and line bisectors.

Based on previous work, we investigated the following geometries $i$ ) a disc with a spot, an arm, an oscillating arm (stellar wind focused by a close compact companion), ii) a symbiotic star, iii) cool star with a wind and hot compact companion (a possible disc around it), iv) pulsations, and $v$ ) a disc with a dust ring (Fig. 1, right panel).

Our observations defined restrictions on the V2028 Cyg model. The peak of the line must be shifted redward. Radial velocities and bisectors vary differently in the wings 

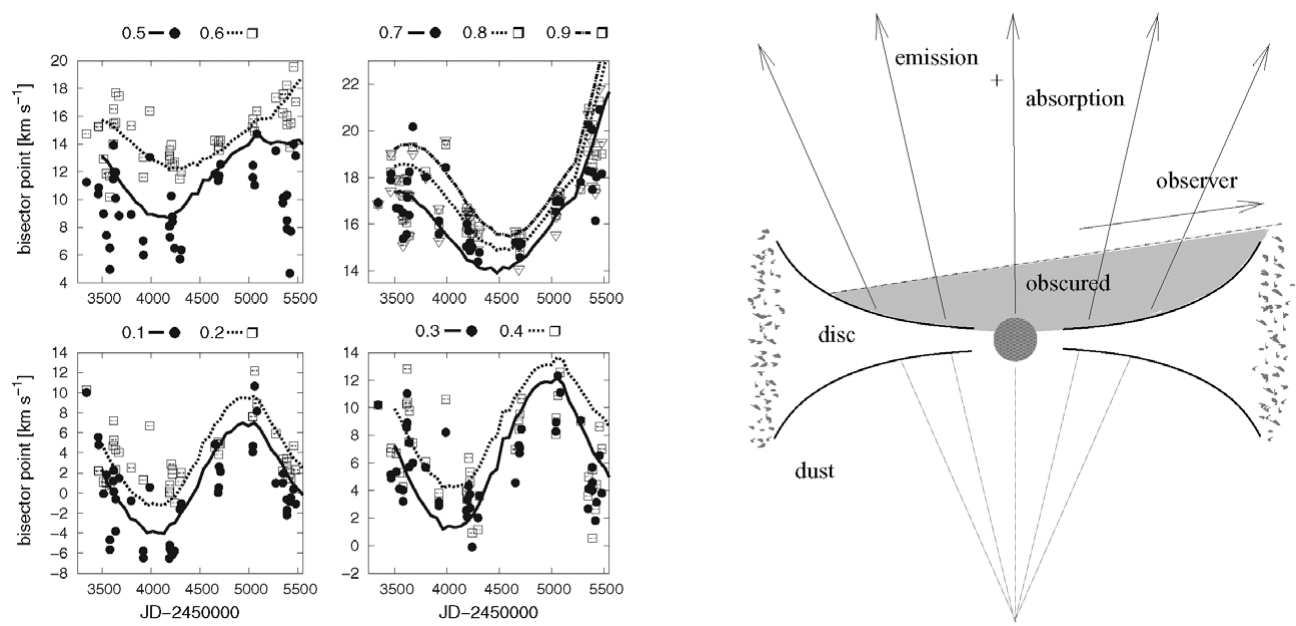

Figure 1. Model corresponding to the observations. The measured (points) and fitted (lines) $\mathrm{H} \alpha$ line bisectors are plotted for the relative highs 0.1 (wings) -0.9 (peak).

and peak. The absolute value of the equivalent width is correlated with the maximum of relative flux and anti-correlated with the radial velocity of the wings.

\section{Results and conclusion}

It is possible to fit the line-profile shape with all the models, however, the observed time dependencies agreed only with a star surrounded by a gaseous disc with a dust ring. The best fit is plotted in Fig. 1 (left panel). The disc with a dust ring must obscure a significant part of the disc itself, otherwise the dependencies will be broken.

The resulting model of the disc with the dust ring implies an alternative explanation of the two-component spectra of V2028 Cyg. The spectrum with features of a hot star originates in the internal part of the disc and wind region. The outer parts of the disc, which we see edge-on, have low temperatures close to the dust condensation value. These conditions allow the formation of an absorption spectrum of the $\mathrm{K}$ type.

In order to verify this hypothesis, we compared the radial velocity measurements of the $\mathrm{H} \alpha$ line and the absorption $\mathrm{K}$ component. We found a similar trend in these dependencies (Polster et al. 2011a). This supports the hypothesis of the geometrically thick disc. However, the accuracy of our measurements is low. There still remains the possibility, that V2028 Cyg is a binary. Considering earlier work (Zickgraf 2001) and our observations, the orbital period of the system in this case must be larger than 25 years.

\section{Acknowledgements}

This research is supported by grants 205/09/P476 (GA ČR), 205/08/H005 (GA ČR), MUNI/A/0968/2009, and projects MSM0021620860 (MŠMT ČR) and AV0Z10030501 (AV ČR).

\section{References}

Bergner, Yu. K., Miroshnichenko, A. S., Yudin, et al. 1995, A\&AS, 112, 221

Polster, J., D. Korčáková, V. Votruba et al., 2010, ASP-CS, 435, 399

Polster, J., D. Korčáková, V. Votruba et al., 2011a, A\&SA, in preparation

Polster, J., D. Korčáková, V. Votruba et al., 2011b, in IAU Symposium 272, in press

Schneider, D. P. \& Young, P. 1980, ApJ, 238, 946

Zickgraf, F. J. 2001, A\&GA, 375, 122 